\title{
A Novel Zero Velocity Detection Method Based on the Plantar Pressure
}

\author{
Chengbin Wang ${ }^{1,2}$, Meifeng Guo ${ }^{1, *}$, Juan Wu ${ }^{2}$, Bin Zhou ${ }^{1}$ and Qi Wei ${ }^{1}$ \\ 1 Engineering Research Center for Navigation Technology, Department of Precision Instruments, Tsinghua \\ University, Beijing100084, China; wcb17@mails.tsinghua.edu.cn(C.W.); \\ guomf@mail.tsinghua.edu.cn(M.G.); zhoub@mail.tsinghua.edu.cn(B.Z.); weiqi@tsinghua.edu.cn(Q.W.) \\ 2 Xi'an Research Institute of Surveying and Mapping, Xi'an 710054, China; juanwu706@gmail.com(J.W.) \\ * Correspondence: guomf@mail.tsinghua.edu.cn; Tel.: +86-10- 6279-2119
}

\begin{abstract}
The zero velocity update(ZUPT) algorithm is the core of a foot-mounted pedestrian navigation system. To make ZUPT work properly, it is necessary to detect zero velocity intervals correctly. We present a study on the zero velocity detection method based on the plantar pressure. In this study, we used the plantar pressure of the forefoot and heel to make a combination of zero velocity detection, which has higher detection accuracy and better environmental adaptability. First, the paper analyzes the motion characteristics of foot during walking. Second, the inherent relationship between the plantar pressure and the gait change during walking is studied based on the pressure sensor. Then, the model of the zero velocity detection method using the plantar pressure is established. Finally, the multi-scene experiments show that this method can improve the accuracy of detection by $10 \%$ on average in outdoor walking environments.
\end{abstract}

Keywords: Pedestrian navigation; ZUPT; Zero Velocity Detection; Plantar Pressure

\section{Introduction}

Location information is closely related to people's daily life. Real-time acquisition of pedestrian location is conducive to the refinement and accuracy of location services. Moreover, in some scenarios (fire control, supervision, security and protection, etc.), real-time acquisition of pedestrian location is crucial [1]. However, the acquisition of pedestrian location information is different from the positioning of a moving platform (car, airplane, ship), the portability of the pedestrian should be considered. With the development of micro-IMU technology, wearable pedestrian positioning technology has developed rapidly, which can effectively solve the problem of pedestrian positioning when GNSS signal is blocked in urban, indoor, underground, forest and other scenes [2]. The foot-mounted pedestrian navigation technology based on human walking gait is a hot topic for many scholars [3-7].The core method is to suppress the error divergence of MIMU devices and improve the accuracy of pedestrian positioning by identifying the movement state of foot in walking and taking advantage of the characteristic that the sole of foot is stationary on the ground. This is also a common method named ZUPT in pedestrian positioning [8-12]. However, the effective use of this method is based on the premise of accurate and timely judgment of human walking gait characteristics. The ZUPT method is the core of foot-mounted pedestrian navigation technology, while the zero-velocity detection method is the premise and guarantee for the correct use of ZUPT. Many scholars have carried out relevant studies on the detection methods of zero velocity of foot in walking.

The first kind of method is to compare the statistical characteristics (amplitude and variance) of the IMU device's output signal with the preset threshold value in the process of human walking. Skog et al. [13-14] compared four zeros velocity detection methods(MV,MAG,ARE,SHOE), using the acceleration moving variance, the acceleration magnitude, the angular rate energy, and the synthesis of them. Yan Li et al. [15] used both accelerometer and gyro measurements in real-time conditions, 
and designed four constraints and six thresholds for detection. Wang Pu et al. [16] used the acceleration variance and the sum of the squares of the gyro modulus value for the detection. Zhang R. et al. [17] proposed a new detection method using an additional chest-attached accelerometer to update corresponding threshold for the zero velocity detection of foot-mounted pedestrian navigation system. These zero velocity detection methods are simple and easy to use, but they are subject to the influence of detection threshold setting. Especially by the difference of devices, users, walking speed and environment, the accuracy of detection is greatly affected.

The second method is to use the neural network, deep learning and other methods for detection. Park et al. [18] proposed a zero velocity detection method, where only one gyroscope value is used, and a Markov model is constructed using segmentation of gyroscope outputs instead of using gyroscope outputs directly. Qiu et al. [19] used the k-mean clustering algorithm to estimate the adaptive time constraint parameters to eliminate the false stance phase detection. $\mathrm{Xu}$ et al. [20] proposed a robust zero velocity detector algorithm using a Bayesian Network model based on the measurements of inertial sensors. Sun et al. [21] thought that it was difficult to detect walking gaits with a fixed threshold method, and proposed a method based on a hidden Markov model using the measurement of a single gyro output. Wagstaff et al. [22] proposed a detection method with a long short-term memory neural network. Although these methods can accurately estimate the gait characteristics of walking foot, but they still have great limitations in real-time computing and embedded system application.

Some other scholars have noticed the natural characteristics of foot when walking. Due to the gravity and walking speed of people themselves, when the foot is still relative to the ground, the sole of the foot will contact the ground and generate pressure. By measuring the pressure information between the sole of the foot and the ground, the movement state of the foot can be detected. Skog et al. [14] used the pressure sensitive resistances to measure the pressure of the soles of foot during walking as a reference standard for zero velocity detection, but it was not considered as a detection method. Bebek et al. [3] proposed a detection method using a high-resolution thin flexible error-correcting biomechanical ground reaction sensor cluster(GRSC), but this paper focused on the design and analysis of the sensor itself. Guo et al. [23] used the ground reaction sensor array(GRSA) to detect the zero velocity of foot during walking, analyzing the influence of GRSA size and resolution on zero-speed detection method, but the detection algorithm was not studied in detail. Ma et al. [24] used the measurements of an accelerometer, gyroscope and pressure sensor to construct a zero-velocity detector, but only the pressure characteristics of the heel was considered, and there was also the problem of threshold setting.

In this paper, we propose a novel zero velocity detection method using the plantar pressure. Based on the variation characteristics of plantar pressure and gait in walking, we established a combined detection model of plantar pressure, and the zero velocity detection method of plantar pressure is implemented, which has higher detection accuracy and better environmental adaptability.

The key contributions of our work are as follows:

- We analyze the relationship between plantar pressure and gait by visual synchronization. This is helpful to obtain the characteristics of plantar pressure changes during walking.

- We use the pressure of the whole foot to detect, rather than the pressure on the heel or sole alone. This can make the detection results more accurate for each movement state of the foot when walking.

- Although using the pressure of the whole foot to detect can be more complicated, but we give a specific detection model which can be used to guide engineering application.

The layout of the paper is as follows. In Section II, we analyze the gait characteristics of walking according to the problem. Thereafter, in Section III, we analyze the shortcomings of the traditional zero velocity detection methods and the variation characteristics of the plantar pressure and propose the novel method. In Section IV, we evaluate the performance of the detector by multi-scene experiments. Finally, in Section VI, we summarize the results and draw conclusions.

\section{Zero Velocity Detection}




\subsection{Problem Analysis}

For foot-mounted pedestrian navigation system, its advantages lie in two aspects. The first is portability. The miniature navigation equipment attached to the shoes or embedded in the shoes has less interference with the walking of people. The second is the improvement of pedestrian navigation positioning accuracy. The research of many scholars has shown that [25]-[29] the ZUPT algorithm can greatly improve the error divergence of MIMU inertial devices and improve the positioning accuracy and stability of the system by utilizing the dynamic and static characteristics of the foot during walking.

However, the advantage of using ZUPT algorithm depends on the correct detection of the zero velocity state and the correct judgment of the zero velocity duration. There are two kinds of errors in zero velocity state detection. One is to think of the dynamic state as static state, and the other is to think of the static state as dynamic state. The first kind of detection error will directly cause the increase of the pedestrian navigation positioning error. The second kind of detection error will not directly increase the positioning error, but the error convergence time of MIMU inertial devices will be reduced and this will affect improvement of positioning.

Therefore, in order to make ZUPT algorithm work effectively in improving the performance of foot-mounted pedestrian navigation system and meet the needs of pedestrian navigation system in different environments, we need to find a zero velocity detection method with higher environmental adaptability and robustness.

\subsection{Analysis of Foot Movement}

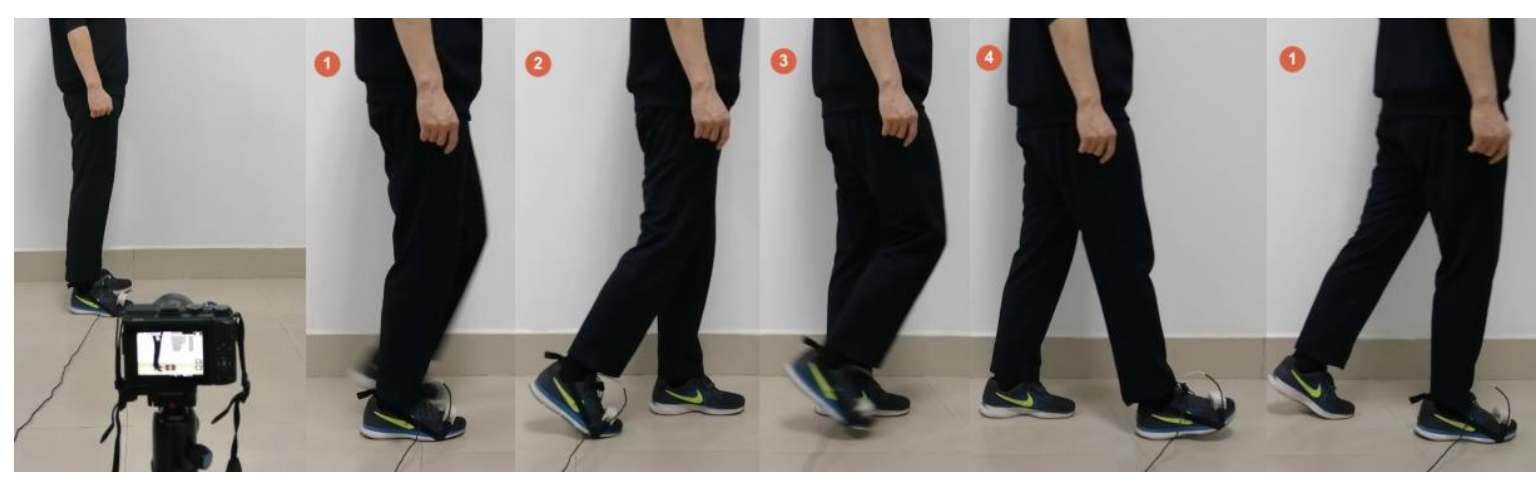

Figure 1. The four stages of the walking gait cycle.

In the process of walking, due to physiological characteristics, the foot movement state relative to the ground is in a process of dynamic and static alternation $[7,8,10]$. In order to analyze the characteristics of foot movement more clearly, we filmed the complete movement state of foot during walking with a camera. We used the time synchronization pulse to realize the time synchronization of video frame, IMU and plantar pressure sensor data. The time resolution can reach $1 \mathrm{~ms}$, which meets the needs of foot motion characteristic analysis.

In Figure 1, there is a complete gait cycle of foot movement, which can be roughly divided into four stages:

(1) The stage of foot static, it is the main static state of the foot. In this stage the whole sole of the foot is attached to the ground relatively static (the (1) of Figure 1);

(2) The stage of foot raising, it is the beginning of the step and is the transformation from static state to dynamic state. This stage lasts from the heel up to the toe off the ground (the (2) of Figure 1);

(3) The stage of foot swing, it is the main dynamic state of the foot. In this stage the foot moves forward as the body's center of gravity moves (the (3) of Figure 1);

(4) The stage of foot landing, it is the ending of the step and is the transformation from dynamic state to static state. This stage lasts from the heel landing to the toe landing (the (4) of Figure 1).

In these four stages, only the stage (1) is useful for error correction where we can use the ZUPT method, and it needs to be accurately detected. Zhao et al. [30] proposed a dual-gait zero velocity 
detection method, dividing the gait cycle to two phases, a stance (4) and (1) and a swing (2) and (3)). But from the analysis of our experimental results, the stage of (4) will introduce the additional errors in the process of INS calculation when assuming the stage (4) to be stance. So we need to detect the stage (1) accurately.

In the process of pedestrian navigation experiment, we found that when zero velocity detection was carried out by using the statistical characteristics of the output signals of IMU gyro and accelerometer, the detection accuracy would decline due to the difference between the user (height, weight, etc.) and the use scene (level of the ground). We also found that for the detection of stage (2) and stage (4), the "quasi-dynamic" and "quasi-static" characteristics are more likely to cause detection error. This can be improved by adjusting the judgment threshold and time window parameters, but is not easy to apply in practice.

Through further analysis of the characteristics of human gait in the process of walking, we found that although the physiological characteristics of people are different and the walking environment is different, but the characteristics of the ability of foot to move the body's center of gravity forward is completely consistent. The forward movement of the center of gravity and the up and down movement of the center of gravity will be reflected in the plantar pressure when walking. Therefore, the movement state of the foot can be truly reflected through the plantar pressure. The forward and up-and-down movement of the body's center of gravity will reflect the plantar pressure. Therefore, the plantar pressure can truly reflect the movement state of the foot.

\section{A Novel Zero Velocity Detector}

\subsection{Data Collection}

In order to qualitatively analyze the relationship between the characteristics of foot pressure change and foot movement state, and to facilitate the comparative analysis with the traditional detection algorithm, we designed a set of data acquisition scheme. The scheme (Figure 2) adopts the micro-inertial pedestrian navigation system developed by the laboratory, including 3 MEMS gyros and 3 MEMS accelerometers, which are strapped to the front foot palm. The coordinates of the IMU are $\mathrm{x}$ axis to the right, $\mathrm{y}$ axis to the front, and $\mathrm{z}$ axis to the top.

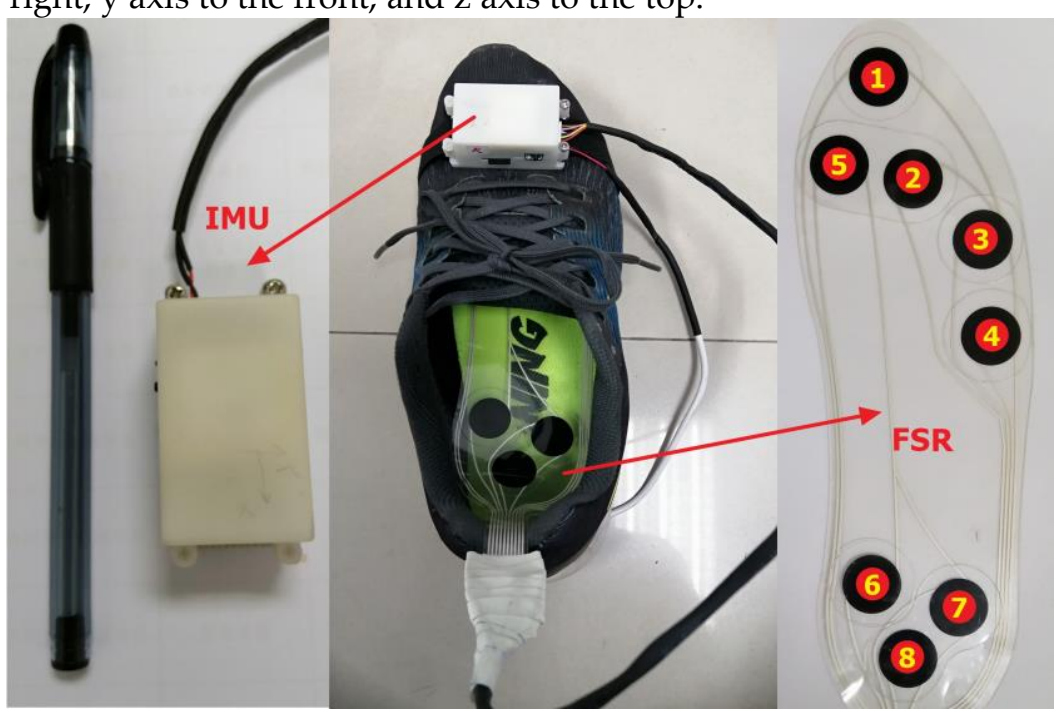

Figure 2. The scheme of data collection.

In order to collect the data of plantar pressure, we used an insole sensor with 8 force-sensitive resistors (FSR). The FSR was developed by using a novel nanomaterial based on the principle that changes in surface pressure cause changes in its own resistance. By measuring the change of its resistance value through the peripheral circuit, we can obtain the pressure value on the sensor. We consider the differences of the walking habits of different people and the distribution of force on the sole of the foot is not consistent, like some people's plantar pressure are inwards, the others are 
outwards. In the scheme, we collected the pressure values of 8 points on the sole of the foot at one time.

In order to ensure the correctness of data comparison, we aligned the IMU data with the planter pressure data during the process of data collection by using unified time pulses to trigger data collection. The data sampling rate is $200 \mathrm{~Hz}$. Two volunteers were selected for the preliminary experiment: a female with height of $165 \mathrm{~cm}$ and weight of $50 \mathrm{~kg}$, a male with height of $173 \mathrm{~cm}$ and weight of $65 \mathrm{~kg}$. We selected two representative experimental scenarios (In Figure3), scene (a) is a plastic playground on the campus where is very flat and scene (b) is an uneven dirt road in the forest.

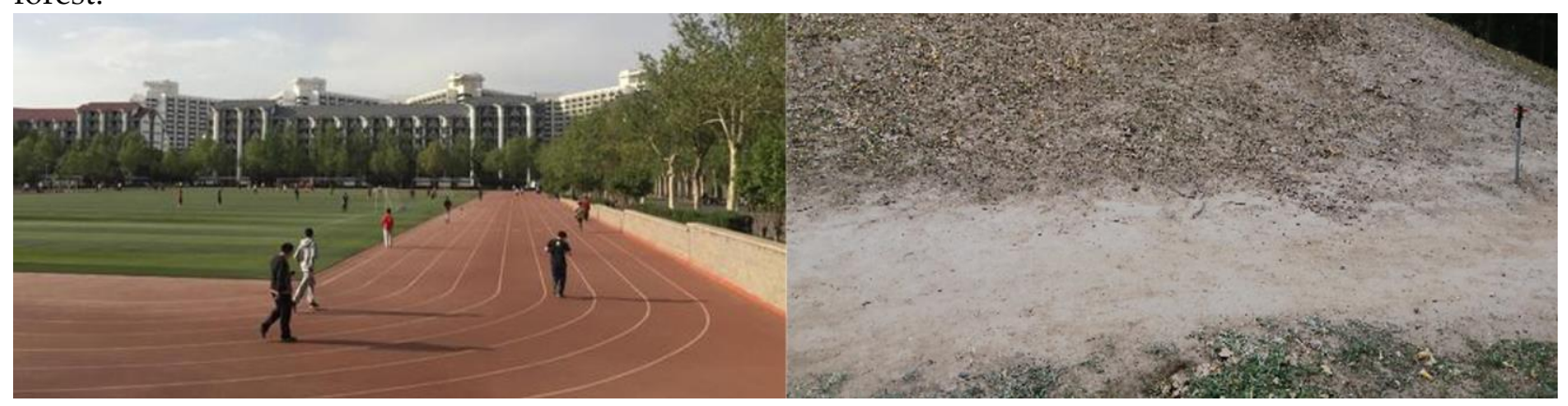

(a) The scene of plastic playground

(b) The scene of uneven dirt road

Figure 3. The experimental scenarios include a plastic playground (a) and an uneven dirt road (b).

\subsection{IMU Data Analysis}
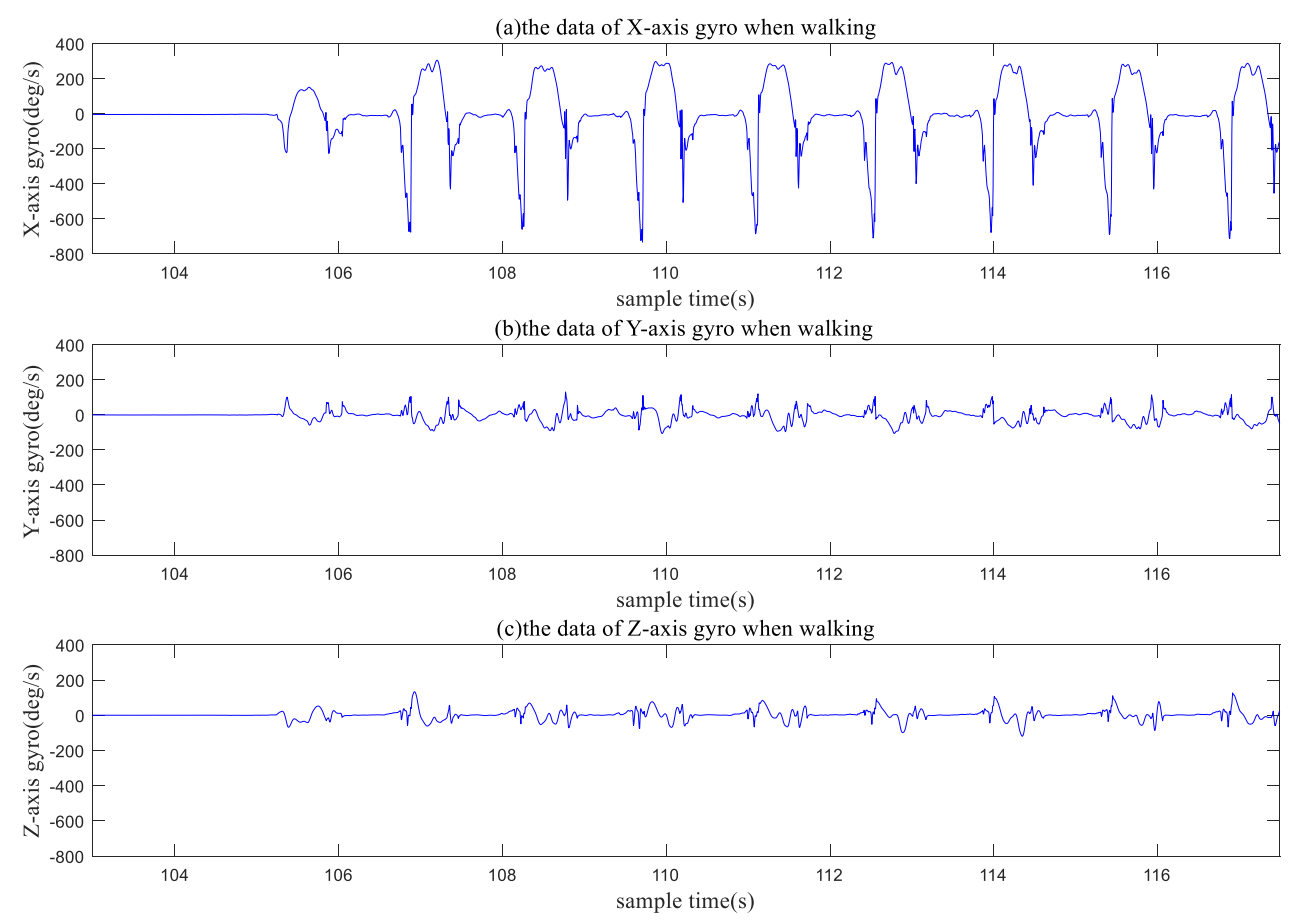

Figure 4. The gyros output data of the male tester walking in scene A. 
We first analyze the collected IMU gait data. In Figure 4, there are the gyros output data of the male experimenter walking in scene A. In Figure 5, there are the accelerometers output data of the male experimenter walking in scene A. From the overview of the gyros and accelerometers output, it can be clearly seen that the IMU data changes with the foot movement state during walking. Obviously, the X-axis gyro and $\mathrm{Y}$-axis accelerometer have the biggest change range and the most obvious trend to the movement of the foot during walking. This also accords with the physiological characteristics of people in the process of walking, lifting and landing the foot when the rotation movement of the ankle and a forward kick. When selecting IMU data as the input of zero velocity detection method, most scholars also choose X-axis gyro and Y-axis accelerometer to calculate.
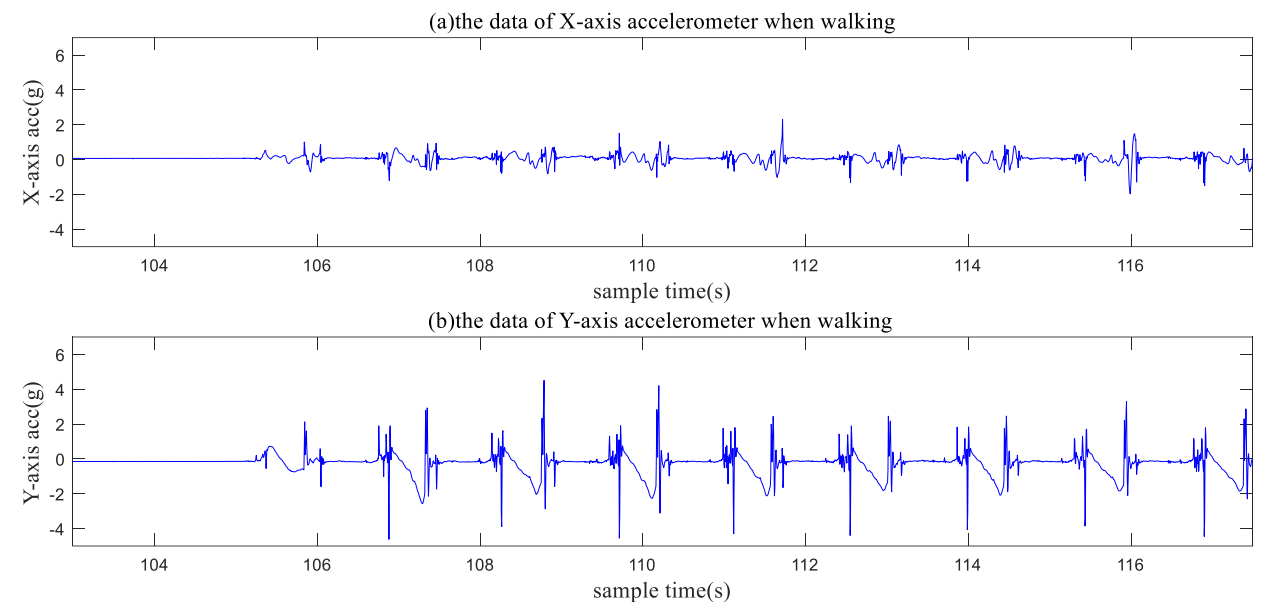

(c)the data of Z-axis accelerometer when walking

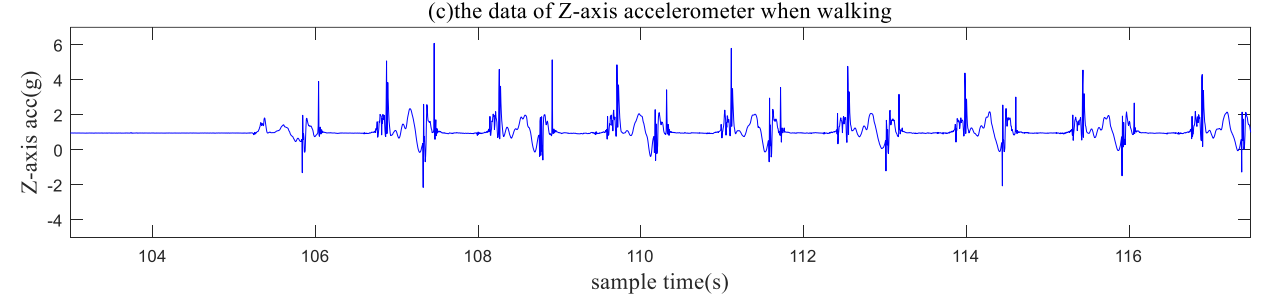

Figure 5. The accelerometers output data of the male tester walking in scene A.

The traditional zero velocity detection method is used to analyze the collected data, and the method model is established first. For IMU sensors, the IMU measurement can be described as follows:

$$
\begin{aligned}
& \mathbf{y}_{k}=\mathbf{s}_{k}+\mathbf{v}_{k} \\
& \mathbf{s}_{k}=\left[\begin{array}{c}
\mathbf{s}_{k}^{a} \\
\mathbf{s}_{k}^{w}
\end{array}\right] \\
& \mathbf{v}_{k}=\left[\begin{array}{c}
\mathbf{v}_{k}^{a} \\
\mathbf{v}_{k}^{w}
\end{array}\right]
\end{aligned}
$$

where $\mathbf{s}_{k}^{a} \in \mathbb{R}^{3} 、 \mathbf{s}_{k}^{w} \in \mathbb{R}^{3}$ denote the IMU true measurement of acceleration and the angular rate at time $\mathrm{k}$, respectively. Moreover, $\mathbf{v}_{k}^{a} \in \mathbb{R}^{3} 、 \mathbf{v}_{k}^{w} \in \mathbb{R}^{3}$ represent the measurement noise of the accelerometers and gyros respectively. We assume that the measurement noise is independent, identically-distributed white Gaussian noise with the covariance matrix.

$$
\mathbf{C}=\mathbf{E}\left[\mathbf{v}_{k} \mathbf{v}_{k}^{T}\right]=\left[\begin{array}{cc}
\sigma_{a}^{2} \mathbf{I}_{3} & \mathbf{0}_{3} \\
\mathbf{0}_{3} & \sigma_{w}^{2} \mathbf{I}_{3}
\end{array}\right]
$$

where $\mathbf{I}_{3}\left(\mathbf{0}_{3}\right)$ denotes an identity(a zeros) matrix of three orders, the $T$ denotes the transpose. To simplify the processing, it is assumed that the three-axis accelerometers and gyros have the same noise characteristics, $\sigma_{a}^{2} \in \mathbb{R}^{1}$ and $\sigma_{w}^{2} \in \mathbb{R}^{1}$ denotes the noise variance. 
The zero velocity detection method using the data of IMU could be formalized as a binary hypothesis testing problem, where the detector can choose between the two hypotheses $H_{0}$ and $H_{1}$ :

$$
\begin{aligned}
& H_{0}: \text { IMU is non- stationary } \\
& H_{1} \text { : IMU is stationary }
\end{aligned}
$$

The determinate criterion is:

$$
\begin{aligned}
& H_{0}: \exists k \in \Omega_{n}, \text { s.t. } \mathbf{s}_{k}^{a} \neq \text { g } \mathbf{u}_{n} \text { or } \mathbf{s}_{k}^{w} \neq 0 \\
& H_{1}: \forall k \in \Omega_{n}, \text { then } \mathbf{s}_{k}^{a}=g \mathbf{u}_{n} \text { and } \mathbf{s}_{k}^{w}=0
\end{aligned}
$$

where $\Omega_{n}=\{\ell \in \mathbb{N}: n \leq \ell<N-1\}$ is the time window parameter required for detection, $N$ is the sampling length of the time window. $\mathbf{u}_{n} \in \mathbb{R}^{3},\left\|\mathbf{u}_{n}\right\|=1$ denotes the unit vector of gravity at rest. By transformation, we can rewrite the criterion as the zero velocity detection operator:

$$
\frac{1}{N} \sum_{k \in \Omega_{n}}\left(\frac{1}{\sigma_{a}^{2}}\left\|\mathbf{S}_{k}-g \frac{\overline{\mathbf{s}}_{n}}{\left\|\overline{\mathbf{s}}_{n}\right\|}\right\|^{2}+\frac{1}{\sigma_{w}^{2}}\left\|\mathbf{s}_{k}^{w}\right\|^{2}\right)<\gamma^{\prime}
$$

where $\gamma^{\prime}=-2(\operatorname{In} \gamma) / N, \gamma$ Is the judgment probability calculated according to the binary hypothesis.

Based on the above model, we first collected data from tester A in the static state after wearing the device, and established the corresponding judgment threshold parameters by analyzing the statistical characteristics of IMU data. By using the threshold parameters, we analyzed the gait characteristics of the two experimenters walking in the two scenarios, and the gait detection results

\begin{tabular}{|c|c|c|c|}
\hline \multicolumn{4}{|c|}{ Tester A: female、 $165 \mathrm{~cm} 、 50 \mathrm{~kg}$} \\
\hline \multicolumn{4}{|c|}{ Scene A: a plastic playground } \\
\hline Number & Walk steps & Detect steps & Accuracy \\
\hline 1 & 243 & 235 & $96 \%$ \\
\hline 2 & 237 & 233 & $98 \%$ \\
\hline 3 & 246 & 232 & $94 \%$ \\
\hline 4 & 465 & 453 & $97 \%$ \\
\hline 5 & 458 & 448 & $97 \%$ \\
\hline \multicolumn{4}{|c|}{ Scene B: an uneven dirt road } \\
\hline Number & Walk steps & Detect steps & Accuracy \\
\hline 1 & 172 & 153 & $89 \%$ \\
\hline 2 & 167 & 154 & $92 \%$ \\
\hline 3 & 175 & 165 & $94 \%$ \\
\hline 4 & 174 & 163 & $94 \%$ \\
\hline 5 & 168 & 153 & $91 \%$ \\
\hline
\end{tabular}
are shown in Table 1 and Table 2. It can be seen from the results that when the threshold judgment method is used for zero velocity detection, the uncertainty of detection accuracy will be caused in different users and different scenarios due to the limitation of using the threshold.

Table 1. The result of tester A on scenes.

Table 2. The result of tester B on scenes.

\begin{tabular}{c|c|c|c}
\hline \multicolumn{4}{c}{ Tester B: male、173cm、65kg } \\
\hline \multicolumn{4}{c}{ Scene A: a plastic playground } \\
\hline Number & Walk steps & Detect steps & Accuracy \\
\hline 1 & 193 & 175 & $91 \%$ \\
\hline 2 & 183 & 162 & $89 \%$ \\
\hline 3 & 197 & 173 & $88 \%$ \\
\hline 4 & 369 & 302 & $82 \%$ \\
\hline 5 & 371 & 330 & $89 \%$ \\
\hline
\end{tabular}




\begin{tabular}{c|c|c|c}
\hline \multicolumn{4}{c}{ Scene B: an uneven dirt road } \\
\hline Number & Walk steps & Detect steps & Accuracy \\
\hline 1 & 133 & 105.07 & $79 \%$ \\
\hline 2 & 129 & 108.36 & $84 \%$ \\
\hline 3 & 137 & 116.45 & $85 \%$ \\
\hline 4 & 136 & 110.16 & $81 \%$ \\
\hline 5 & 130 & 114.4 & $88 \%$ \\
\hline
\end{tabular}

From the above table, we can see that the detection accuracy decreases when the walking scene changes for tester A, which is mainly due to that the road of scene B was potholed. When people walk on the uneven road, the sole of their feet will be affected by additional vibration, which will cause the IMU to vibrate and the statistical properties of the output data will be changed. This is also the main reason why the detection accuracy decreases with the change of scenes.

In addition, we also found another phenomenon, when the experimenter changed, the accuracy of the zero velocity detection decreased in both scenarios. In order to analyze the reasons for the decline in detection accuracy, a section of data of tester B in scene B was selected for detailed analysis.

The output data of $\mathrm{Y}$-axis accelerometer and $\mathrm{X}$-axis gyro with the most obvious dynamic characteristics during walking were selected for analysis. We extracted the static data of tester $B$ in the process of walking in scene B according to the starting and ending moments of the static state judged by the SHOE detection method. All the data judged to be in a static state were combined and plotted as shown in Figure 6. The first flat data of the figure is the static data collected when the foot stand still before the start of walking, and the data in different colors after that are the data collected when the foot are stationary at each step of walking.
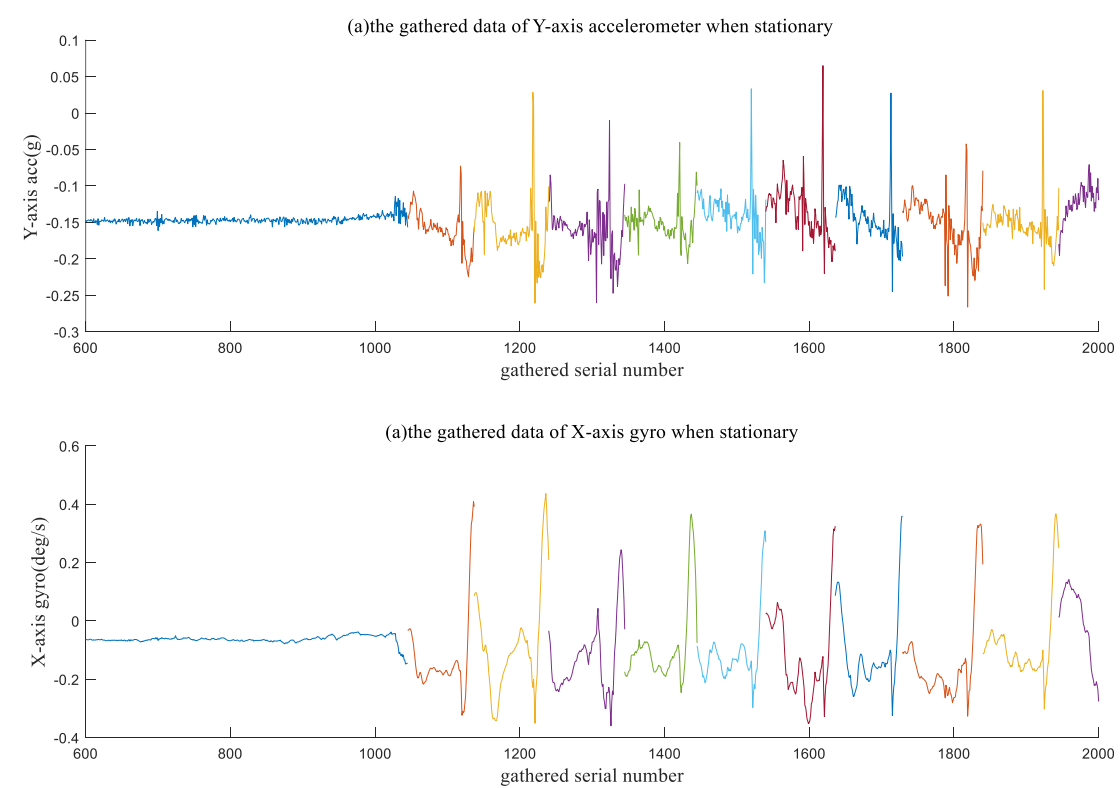

Figure 6. The gathered data of $\mathrm{Y}$-axis accelerometer and the $\mathrm{X}$-axis gyro when stationary of the male experimenter walking in scene B.

From the figure, we can see that when the foot stands still at the beginning, the output data of the device is relatively flat, which conforms to the signal statistical characteristics in the static state. However, although the variation amplitude of the output signal in the static state during walking is small and nearly flat (as shown in Figure 5), the variation amplitude of the signal is still large, and some has exceeded the judgment threshold parameters. This is because the stationary state of foot on walking is not as stable as the stationary state of standing. There will be a small vibration or shaking on foot. And this will cause the fluctuations in the IMU signal, and at some time the preset threshold parameters no longer apply. Through experiments, the detection accuracy of different users can be 
improved to a certain extent by adjusting threshold parameters or the length of detection time, but it is not convenient for practical application.

\subsection{Plantar Pressure Analysis}

In order to study the relationship between plantar pressure and walking gait, the video information of foot movement and foot pressure during walking were collected through time synchronization, and then compared and analyzed. In the Figure 7, the plantar pressure change information in two walking gait cycles was plotted. In order to facilitate the display, only the pressure information at the stress point 5 of the forefoot and the pressure point 8 of the heel were selected.

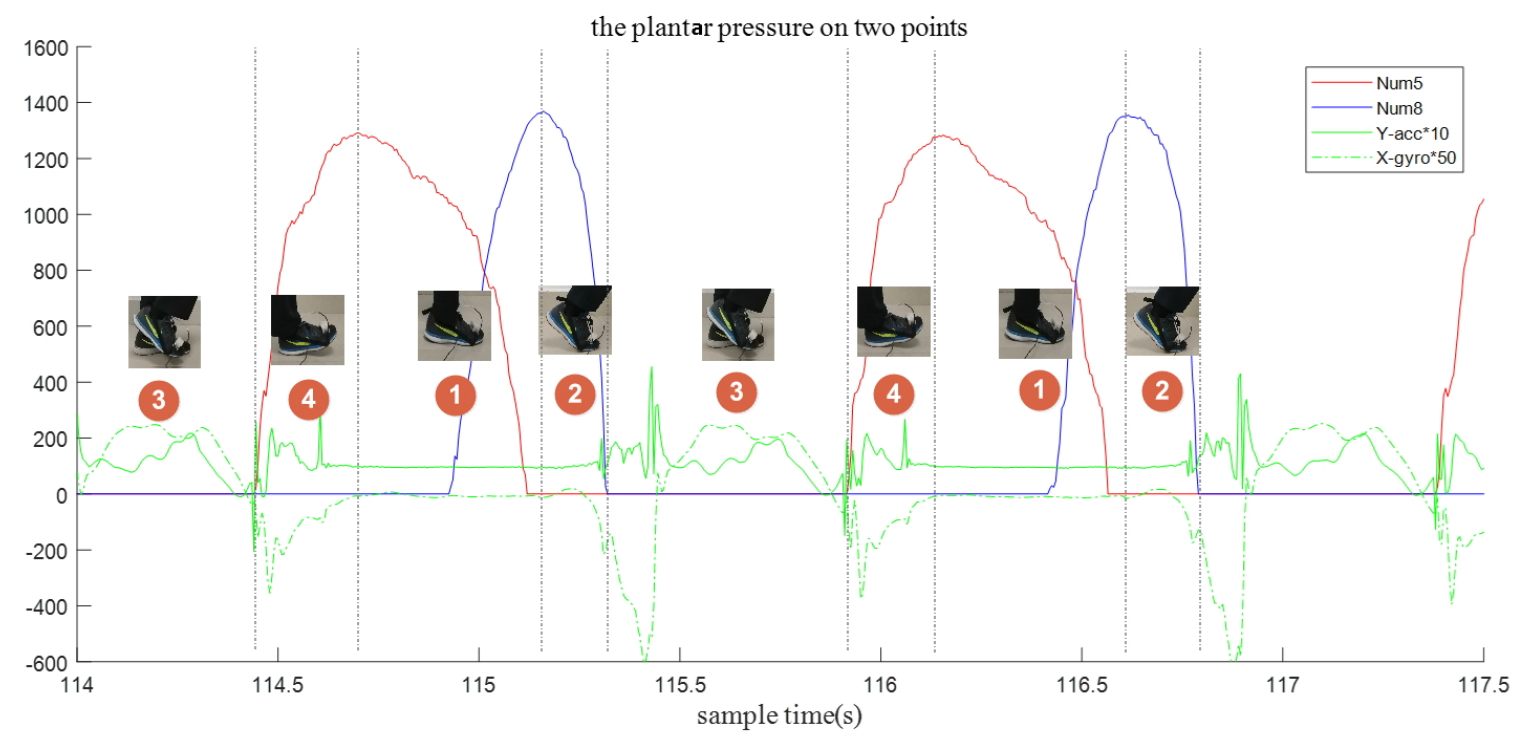

Figure 7. The characteristics of plantar pressure during walking.

In the Figure 7 , the data information of the $\mathrm{Y}$-axis accelerometer and the $\mathrm{X}$-axis gyro of the IMU is also drawn. In order to facilitate comparison, the output of the $\mathrm{Y}$-axis accelerometer and the $\mathrm{X}$-axis gyro is amplified by 10 and 50 times respectively. According to the analysis of plantar pressure data using the time-synchronized video as a reference, it can be found that during a walking gait cycle, there is a clear corresponding relationship between plantar pressure and the four stages of gait:

(1) When the foot is in the movement phase (3), the phenomenon is: the foot is swinging in the air, the sole of the foot is not in contact with the ground and the plantar pressure is zero, the FSR has no output, the red line and the blue line in the figure are approximately zero.

(2) When the foot is in the movement phase (4), the changing process of foot movement is as follows: first, the heel touches the ground; second, the sole rotates with the heel as the axis and landing on the ground. The pressure on the heel gradually increases with the forward movement of the body's center of gravity, until the body's center of gravity is completely on the heel and reaches the maximum. At this moment, the signal output of accelerometer and gyro (the green curve in the Figure 7) also tends to be flat from oscillation, which also conforms to the motion characteristics of the foot.

(3) When the foot is in the movement phase (1), the sole of foot is in full contact with the ground and support the body's center of gravity. In this stage, there is an obvious feature, which is shown in the Figure 7 that the pressure value of the heel (Num5) drops from the peak to 0 , while the pressure value of the sole of the foot (Num8) rises from 0 to the maximum. This change also means that the center of gravity of the human body in the process of walking, from heel support to foot support is line with the physiological characteristics of human walking.

(4) When the foot is in the movement phase (2), the body's center of gravity is off the bottom of the foot and the heel is raised without pressure. The pressure on the sole of the foot dropped rapidly from its peak to zero, as shown in the blue curve in phase (2). The output 
of the accelerometer and gyro also changes from flat to oscillating (the green curve in the figure) until the entire foot is off the ground and into the next phase of motion.

After completing a gait cycle in this way, it can be found through these analyses that zero velocity detection of gait can be carried out during walking by using the pressure value variation characteristics at the ball of the foot and the heel of the sole.

\subsection{Detection Method}

Based on the analysis of characteristics of gait changes with plantar pressure, a zero velocity detection model based on the characteristics of plantar pressure changes was constructed. We used the off-the-shelf insole FSR sensors in Figure 2 to measure the plantar pressure. Mario et al. [31] used the same type of sensor to research the optimal set of pressure sensors and their locations on a pressure monitoring insole. From our experiment, we found that the distribution of plantar pressure will be different and the variation characteristics of 8 measuring points of sole will be inconsistent for different users, different roads, or even the shoes with different soft and hard texture. From the perspective of the zero velocity detection, we combined the pressure data collected in real time according to the pressure on the sole and the heel. In combination with the distribution of measurement points of the FSR on the sole of the foot, established the signal model as follows:

$$
\begin{aligned}
& p_{f}=p_{1}+p_{2}+p_{3}+p_{4}+p_{5} \\
& p_{b}=p_{6}+p_{7}+p_{8}
\end{aligned}
$$

where $p_{i}(i=1,2,3 \ldots 8)$ denotes the pressure measurement at point $i$ on the FSR in Figure $2, p_{f}$ denotes the total pressure of the five measuring points on the sole, $p_{b}$ denotes the total pressure of the three measuring points on the heel. This can avoid the detection error caused by the different walking habits of different people.

In this method, we design to detect the state of gait based on the pressure of the sole and heel. From the previous analysis, we can see that the pressure peak of the sole and heel can be used as the critical point of the four stages of gait. We use the peak detection algorithm [32] to find the time when the stage changes. The peak detection algorithm as follow:

$$
\begin{aligned}
& \bar{p}_{f}\left(t_{k}\right)=\frac{1}{N} \sum_{i=k-\frac{N}{2}}^{k+\frac{N}{2}} p_{f}\left(t_{i}\right) \\
& \left\{\begin{array}{lr}
\bar{p}_{f}\left(t_{k-1}\right) \leq \bar{p}_{f}\left(t_{k}\right) \& \& \bar{p}_{f}\left(t_{k}\right)>\bar{p}_{f}\left(t_{k+1}\right): & M_{p f}\left(t_{k}\right)=1 \\
\text { others : } & M_{p f}\left(t_{k}\right)=0
\end{array}\right.
\end{aligned}
$$

where $p_{f}\left(t_{i}\right)$ denote the total pressure of the five measuring points on the sole at time $t_{i}$, $N$ denote the time window parameters, $\bar{p}_{f}\left(t_{k}\right)$ denote the mean value of $p_{f}$ at time $t_{k}$, $M_{p f}\left(t_{k}\right)$ denote the peak detection mark, 1 is the peak, 0 is not the peak. But it will be interfered by pseudo peaks.

We use the following rules to determine the true peak:

$$
P_{f}\left(\max , t_{j}\right)=\max _{i=k-\frac{N}{2}}^{i=k+\frac{N}{2}}\left(\bar{p}_{f}\left(t_{k}\right) \mid M_{p f}\left(t_{k}\right)==1\right)
$$

where $P_{f}\left(\max , t_{j}\right)$ denote the peak of the pressure at time $t_{j}$. The calculation method of $P_{b}\left(\max , t_{j}\right)$ is the same $P_{f}\left(\max , t_{j}\right)$.

We designed a zero velocity detection method based on the plantar pressure and the pseudocode is listed in Method 1, where $k$ denotes the data sampling update, State $\in\{0,1\}$ denotes the foot movement, 0 is dynamic and 1 is the stationary. The four phases of foot movement were represented by Stage $\in\{1,2,3,4\}$. The pressure value of the sole when the foot leaves the ground is 
defined as $P_{\min }$, which theoretically should be zero, but due to the hardness of the shoe material and the shoelace is tight or loose, sometimes it is a small value, which can be limited to $(20,50)$.

\section{Method 1 Pseudocode for the zero-velocity detection used plantar pressure}

$1: k=0, N u m=0$

$2:\left\{\right.$ State, Stage,$\left.P_{\min }\right\} \longleftarrow\{$ Initial $\}$

$3:$ loop 1

4: $k=k+1$

5: $\quad p_{f}=\sum_{i=1}^{5} p_{i}(k), p_{b}=\sum_{i=6}^{8} p_{i}(k)$

6: if $p_{b}>P_{\min } \& p_{f}>P_{\min }$

7: $\quad$ Stage $=1$, State $=1$, Num ++

8: $\quad k=k+1$

9: $\quad\left\{p_{f}, p_{b}\right\} \longleftarrow\left\{N e w\left(p_{i}(k)\right)\right\}$

10: $\operatorname{loop} 2\left(p_{b}>P_{\min } \& p_{f}>P_{\min }\right)$

11: $\quad$ Num ++

12: $k=k+1$

13: $\quad\left\{p_{f}, p_{b}\right\} \longleftarrow\left\{N e w\left(p_{i}(k)\right)\right\}$

14: endloop2

15: $\quad$ State $=0$, Num $=0$

16: elseif $p_{b}<P_{\min } \& p_{f}<P_{\min }$

17: $\quad$ Stage $=3$, State $=0$, Num $=0$

18: $k=k+1$

19: $\quad\left\{p_{f}, p_{b}\right\} \longleftarrow\left\{N e w\left(p_{i}(k)\right)\right\}$

20: $\quad \operatorname{loop} 3\left(p_{b}<P_{\min } \& p_{f}<P_{\min }\right)$

21: $k=k+1$

22: $\quad\left\{p_{f}, p_{b}\right\} \longleftarrow\left\{N e w\left(p_{i}(k)\right)\right\}$
23: endloop3

24: elseif $p_{b} \geq P_{\min } \& p_{f}<P_{\min }$

25: $\quad$ Stage $=4$, State $=0$, Num $=0$

26: $\quad \operatorname{loop} 4\left(\left\{p_{b}\right\} \longrightarrow\{\right.$ Maxmun $\left.\}\right)$

27: $\quad k=k+1$

28 :

29:

30 :

$\left\{p_{f}, p_{b}\right\} \longleftarrow\left\{\operatorname{New}\left(p_{i}(k)\right)\right\}$

endloop 4

Stage $=1$, State $=1$, Num $=0$

31:

$$
\operatorname{loop} 5\left(\left\{p_{f}\right\} \longrightarrow\{\text { Maxmun }\}\right)
$$

$k=k+1, N u m++$

$\left\{p_{f}, p_{b}\right\} \longleftarrow\left\{N e w\left(p_{i}(k)\right)\right\}$

endloop 5

34 :

35 :

36 :

else

$$
\text { Stage }=2, \text { State }=0, \text { Num }=0
$$

37 :

38 :

$$
\operatorname{loop} 6\left(\left\{p_{f}\right\} \longrightarrow\left\{P_{\min }\right\}\right)
$$

$$
k=k+1
$$$$
\left\{p_{f}, p_{b}\right\} \longleftarrow\left\{N e w\left(p_{i}(k)\right)\right\}
$$

endloop 6

40 :

41:

end

42: end

43: end

44 : endloop 1

Basically the proposed method works as follows: first, the state information is initialized. Then enter the main loop1, use the data obtained at each sampling time to calculate the $p_{f}$ and $p_{b}$. There are four judgment branches in the main loop1. Using the calculated values of $p_{f}$ and $p_{b}$, we can enter into the judgment of four stages by four detection conditions. After entering each decision branch, variable 'State' is used to identify the current motion state, and variable 'Stage' is used to identify the current gait phase. In each judgment branch, the end condition of the current phase is determined by a self-looping detection method at the sampling time. The maximum value of $p_{f}$ is used to detect the beginning of phase (2) and the maximum value of $p_{b}$ is used to detect the beginning of phase (1).

\section{Experiment and Results}

In order to verify the correctness and environmental adaptability of the zero velocity detection method based on plantar pressure, several representative scenarios were selected for testing. We selected five scenes that cover almost all pedestrian walking environments, including corridor, graveled path, slope, grass land and snow (Figure 8). 


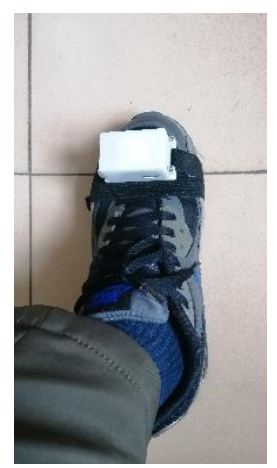

(a) corridor

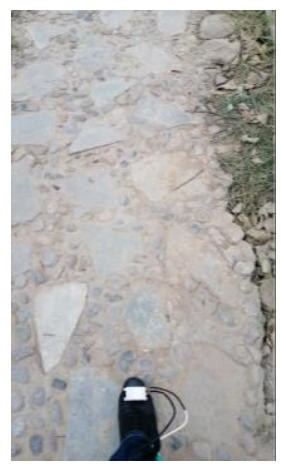

(b)graveled path

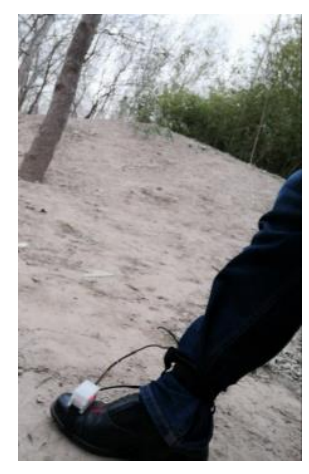

(c)slope

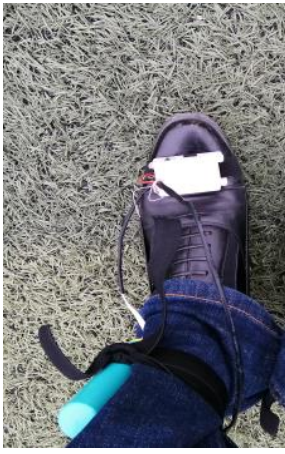

(d)grass land

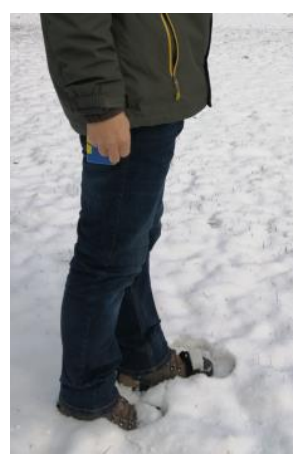

(e)snow

Figure 8. The Multi-scene experiment.

In each scene, the tester (male, $173 \mathrm{~cm}, 65 \mathrm{~kg}$ ) were asked to walk normally for 5 times, and the number of steps in each walk was recorded respectively. Then we compared with the results of the zero velocity detection method, as shown in Table 3 and Table 4 . We used two methods to detect the zero velocity. One is the traditional method and the results as shown in Table III, the other is the planter pressure method and the results as shown in Table IV.

Table 3. The result of detection by traditional method.

\begin{tabular}{c|c|c|c|c|c|c}
\hline \multirow{3}{*}{$\begin{array}{c}\text { Scene } \\
\text { (a) }\end{array}$} & Walk steps & 90 & 89 & 88 & 91 & 90 \\
\cline { 2 - 7 } & Detect steps & 90 & 87 & 87 & 91 & 90 \\
\cline { 2 - 7 } & Accuracy & $100 \%$ & $98 \%$ & $99 \%$ & $100 \%$ & $100 \%$ \\
\hline \multirow{3}{*}{$\begin{array}{c}\text { Scene } \\
\text { (b) }\end{array}$} & Walk steps & 124 & 121 & 123 & 126 & 121 \\
\cline { 2 - 7 } & Detect steps & 110 & 112 & 115 & 114 & 103 \\
\cline { 2 - 7 } & Accuracy & $89 \%$ & $93 \%$ & $93 \%$ & $90 \%$ & $85 \%$ \\
\hline \multirow{2}{*}{$\begin{array}{c}\text { Scene } \\
\text { (c) }\end{array}$} & Walk steps & 76 & 74 & 77 & 77 & 76 \\
\cline { 2 - 7 } & Detect steps & 65 & 66 & 61 & 63 & 70 \\
\cline { 2 - 7 } & Accuracy & $86 \%$ & $89 \%$ & $79 \%$ & $82 \%$ & $92 \%$ \\
\hline \multirow{2}{*}{$\begin{array}{c}\text { Scene } \\
\text { (d) }\end{array}$} & Walk steps & 154 & 149 & 152 & 158 & 155 \\
\cline { 2 - 7 } & Detect steps & 135 & 136 & 139 & 141 & 135 \\
\cline { 2 - 7 } & Accuracy & $88 \%$ & $91 \%$ & $91 \%$ & $89 \%$ & $87 \%$ \\
\hline \multirow{2}{*}{$\begin{array}{c}\text { Scene } \\
\text { (e) }\end{array}$} & Walk steps & 90 & 90 & 89 & 90 & 90 \\
\cline { 2 - 7 } & Detect steps & 80 & 79 & 81 & 80 & 78 \\
\cline { 2 - 7 } & Accuracy & $89 \%$ & $88 \%$ & $91 \%$ & $89 \%$ & $87 \%$ \\
\hline
\end{tabular}

Table 4. The result of detection by plantar pressure.

\begin{tabular}{c|c|c|c|c|c|c}
\hline \multirow{2}{*}{$\begin{array}{c}\text { Scene } \\
\text { (a) }\end{array}$} & Walk steps & 90 & 89 & 88 & 91 & 90 \\
\cline { 2 - 7 } & Detect steps & 90 & 88 & 88 & 90 & 89 \\
\cline { 2 - 7 } & Accuracy & $100 \%$ & $99 \%$ & $100 \%$ & $99 \%$ & $99 \%$ \\
\hline \multirow{3}{*}{$\begin{array}{c}\text { Scene } \\
\text { (b) }\end{array}$} & Walk steps & 124 & 121 & 123 & 126 & 121 \\
\cline { 2 - 7 } & Detect steps & 121 & 118 & 114 & 122 & 117 \\
\cline { 2 - 7 } & Accuracy & $98 \%$ & $98 \%$ & $93 \%$ & $97 \%$ & $97 \%$ \\
\hline \multirow{2}{*}{$\begin{array}{c}\text { Scene } \\
\text { (c) }\end{array}$} & Walk steps & 76 & 74 & 77 & 77 & 76 \\
\cline { 2 - 7 } & Detect steps & 70 & 69 & 70 & 69 & 71 \\
\cline { 2 - 7 } & Accuracy & $92 \%$ & $93 \%$ & $91 \%$ & $90 \%$ & $93 \%$ \\
\hline \multirow{3}{*}{$\begin{array}{c}\text { Scene } \\
\text { (d) }\end{array}$} & Walk steps & 154 & 149 & 152 & 158 & 155 \\
\cline { 2 - 7 } & Detect steps & 143 & 140 & 141 & 150 & 147 \\
\cline { 2 - 7 } & Accuracy & $93 \%$ & $94 \%$ & $93 \%$ & $95 \%$ & $95 \%$ \\
\hline \multirow{2}{*}{$\begin{array}{c}\text { Scene } \\
\text { (e) }\end{array}$} & Walk steps & 90 & 90 & 89 & 90 & 90 \\
\cline { 2 - 7 } & Detect steps & 86 & 85 & 85 & 86 & 86 \\
\cline { 2 - 7 } & Accuracy & $96 \%$ & $94 \%$ & $96 \%$ & $96 \%$ & $96 \%$ \\
\hline
\end{tabular}


Through the comparison and analysis of experimental data in multiple scenarios, we can find that the new detection method has good adaptability to different walking environments, and the detection accuracy can be improved by $10 \%$ on average compared with the traditional method. Moreover, through data analysis, we can also find that:

(1) When the walking ground is flat and hard, the accuracy of the zero velocity detection method based on plantar pressure is higher. This is because the feedback of the floor to the plantar pressure during walking is more timely and accurate, which is conducive to the judgment of gait recognition.

(2) When walking on the uneven gravel road surface, although the uneven ground will have an additional vibration effect on the IMU, it results in a decline in the detection accuracy of the SHOE method (Table III). But the impact on the detection accuracy of the zero-speed detection method of sole pressure is not significant.

(3) When walking on the grass and snow, due to the soft ground, the foot will sink when it falls on the ground to bear the gravity, which will affect the output of the gyro and accelerometer to produce fluctuations, and this will cause SHOE detection errors. But this situation has little impact on the plantar pressure detection method, because the sole of foot will still generate stable pressure during the process of the sinking, which can be used to detect zero velocity.

(4) During the walking test in the slope scene, the accuracy of zero velocity detection of plantar pressure is relatively low. According to the analysis, the reason is that in the process of climbing, the plane inclination between the sole of the foot and the ground is larger than that of walking on the flat ground. The change of the plantar pressure for the movement of the body's center of gravity in climbing is also slightly different from flat walking. Plantar pressure is more focused on the sole than the pressure on the heel, which will cause some deviation in the detection method.

\section{Conclusions}

We presented a novel zero velocity detection method based on the plantar pressure. The inherent relationship between the plantar pressure and gait was analyzed based on the physiological characteristics of walking. The shortcomings of traditional detection algorithm are analyzed through experimental comparison. The zero velocity detection model using plantar pressure is established and the method and work flow were given. The experiment results show that the proposed method not only has a high detection accuracy, but also has a good adaptability to users and walking environment. Of course, there are several avenues for future work. First, in addition to zero velocity detection based on plantar pressure, how to recognize human posture and movement based on plantar pressure, such as sitting, jumping, lying down, falling, etc. Second, how to integrate FSR sensor and IMU on system level for practical application.

\section{Author Contributions:}

Chengbin Wang proposed the original idea and wrote the paper; Bin Zhou and Qi Wei helped to design the experiments; Juan $\mathrm{Wu}$ helped to process the data; Meifeng Guo gave some valuable suggestions and supervised the work.

\section{Conflicts of Interest:}

The authors declare no conflict of interest.

\section{References}

1. Xia, M., et al., Performance Enhancement of Pedestrian Navigation Systems Based on Low-Cost Foot-Mounted MEMS-IMU/Ultrasonic Sensor. Sensors, 2019. 19(2): p. 19.

2. Pham, D.D. and Y.S. Suh, Pedestrian Navigation Using Foot-Mounted Inertial Sensor and LIDAR. Sensors, 2016. 16(1). 
3. Bebek, O., et al., Personal Navigation via High-Resolution Gait-Corrected Inertial Measurement Units. Ieee Transactions on Instrumentation and Measurement, 2010. 59(11): p. 3018-3027.

4. Yun, X.P., et al., Estimation of Human Foot Motion During Normal Walking Using Inertial and Magnetic Sensor Measurements. Ieee Transactions on Instrumentation and Measurement, 2012. 61(7): p. 2059-2072.

5. Duong, P.D. and Y.S. Suh, Foot Pose Estimation Using an Inertial Sensor Unit and Two Distance Sensors. Sensors, 2015. 15(7): p. 15888-15902.

6. Yang, W., et al., A Novel 3D Pedestrian Navigation Method for a Multiple Sensors-Based Foot-Mounted Inertial System. Sensors, 2017. 17(11): p. 30.

7. Zhang, W., D. Wei, and H. Yuan, The Improved Constraint Methods for Foot-Mounted PDR System. Ieee Access, 2020. 8: p. 31764-31779.

8. Wang, Q.Y., et al., Research on the Forward and Reverse Calculation Based on the Adaptive Zero-Velocity Interval Adjustment for the Foot-Mounted Inertial Pedestrian-Positioning System. Sensors, 2018. 18(5): p. 16.

9. Niu, Z., et al., RTK with the Assistance of an IMU-Based Pedestrian Navigation Algorithm for Smartphones. Sensors, 2019. 19(14): p. 25.

10. Zhang, W.C., D.Y. Wei, and H. Yuan, Novel Drift Reduction Methods in Foot-Mounted PDR System. Sensors, 2019. 19(18): p. 28.

11. Poulose, A., O.S. Eyobu, and D.S. Han, An Indoor Position-Estimation Algorithm Using Smartphone IMU Sensor Data. Ieee Access, 2019. 7: p. 11165-11177.

12. Xia, M. and C. Shi, Autonomous Pedestrian Altitude Estimation Inside a Multi-Story Building Assisted by Motion Recognition. Ieee Access, 2020. 8: p. 104718-104727.

13. Skog, I., et al., Zero-Velocity Detection-An Algorithm Evaluation. Ieee Transactions on Biomedical Engineering, 2010. 57(11): p. 2657-2666.

14. Skog, I., J.O. Nilsson, and P. Handel, Evaluation of Zero-Velocity Detectors for Foot-Mounted Inertial Navigation Systems, in 2010 International Conference on Indoor Positioning and Indoor Navigation, R. Mautz, M. Kunz, and H. Ingensand, Editors. 2010, Ieee: New York.

15. Li, Y., et al. A robust humanoid robot navigation algorithm with ZUPT. in Mechatronics and Automation (ICMA), 2012 International Conference on. 2012.

16. Wang, P., et al., Indoor Personal Navigation System Based on Zero Velocity Detection of Pseudo Adaptive Threshold. Journal of Beijing University of Technology, 2015. 41(9): p. 1308-13.

17. Zhang, R., et al., Adaptive Zero Velocity Update Based on Velocity Classification for Pedestrian Tracking. Ieee Sensors Journal, 2017. 17(7): p. 2137-2145.

18. Park, S.K. and Y.S. Suh, A Zero Velocity Detection Algorithm Using Inertial Sensors for Pedestrian Navigation Systems. Sensors, 2010. 10(10): p. 9163-9178.

19. Qiu, S., et al., Inertial/magnetic sensors based pedestrian dead reckoning by means of multi-sensor fusion. Information Fusion, 2018. 39: p. 108-119.

20. Xu, Z.Y., et al., A Robust Method to Detect Zero Velocity for Improved 3D Personal Navigation Using Inertial Sensors. Sensors, 2015. 15(4): p. 7708-7727.

21. Sun, W., et al., Zero velocity interval detection based on a continuous hidden Markov model in micro inertial pedestrian navigation. Measurement Science and Technology, 2018. 29(6).

22. Wagstaff, B., J. Kelly, and Ieee, LSTM-Based Zero-Velocity Detection for Robust Inertial Navigation, in 2018 Ninth International Conference on Indoor Positioning and Indoor Navigation. 2018.

23. Guo, Q.B., et al., Personal Inertial Navigation System Assisted by MEMS Ground Reaction Sensor Array and Interface ASIC for GPS-Denied Environment. Ieee Journal of Solid-State Circuits, 2018. 53(11): p. 3039-3049.

24. Ma, M., et al., An Adaptive Zero Velocity Detection Algorithm Based on Multi-Sensor Fusion for a Pedestrian Navigation System. Sensors, 2018. 18(10): p. 16.

25. Li, Y. and J.J. Wang, A robust pedestrian navigation algorithm with low cost IMU, in 2012 International Conference on Indoor Positioning and Indoor Navigation, C. Rizos, et al., Editors. 2012, Ieee: New York.

26. Guo, Q., et al. A personal navigation system using MEMS-based high-density ground reaction sensor array and inertial measurement unit. in 18th International Conference on Solid-State Sensors, Actuators and Microsystems, TRANSDUCERS 2015, June 21, 2015 - June 25, 2015. 2015. Anchorage, AK, United states: Institute of Electrical and Electronics Engineers Inc.

27. Pham Duy, D. and Y.S. Suh, Foot Pose Estimation Using an Inertial Sensor Unit and Two Distance Sensors. Sensors, 2015. 15(7): p. 15888-15902. 
28. Bao, S.D., et al., Fusion of Inertial/Magnetic Sensor Measurements and Map Information for Pedestrian Tracking. Sensors, 2017. 17(2): p. 17.

29. Song, J.W. and C.G. Park, Enhanced Pedestrian Navigation Based on Course Angle Error Estimation Using Cascaded Kalman Filters. Sensors, 2018. 18(4): p. 20.

30. Zhao, H., et al., Heading Drift Reduction for Foot-Mounted Inertial Navigation System via Multi-Sensor Fusion and Dual-Gait Analysis. Ieee Sensors Journal, 2019. 19(19): p. 8514-8521.

31. Munoz-Organero, M., et al., Sensor Optimization in Smart Insoles for Post-Stroke Gait Asymmetries Using Total Variation and L-1 Distances. Ieee Sensors Journal, 2017. 17(10): p. 3142-3151.

32. Zhang, A.-h., P. Wang, and Y.-x. Chou, Peak detection of pulse signal based on dynamic difference threshold. Journal of Jilin University (Engineering and Technology Edition), 2014. 44(3): p. 847-853. 